\title{
The Least Algebraic Connectivity of Graphs
}

\author{
Guisheng Jiang, ${ }^{1}$ Guidong $\mathrm{Yu}^{2}{ }^{2}$ and Jinde $\mathrm{Cao}^{3,4}$ \\ ${ }^{1}$ School of Physics and Electronic Engineering, Anqing Normal University, Anqing 246011, China \\ ${ }^{2}$ School of Mathematics and Computation Sciences, Anqing Normal University, Anqing 246011, China \\ ${ }^{3}$ Department of Mathematics, Southeast University, Nanjing, Jiangsu 210096, China \\ ${ }^{4}$ Department of Mathematics, Faculty of Science, King Abdulaziz University, Jeddah 21589, Saudi Arabia
}

Correspondence should be addressed to Jinde Cao; jdcao@seu.edu.cn

Received 20 October 2014; Accepted 8 February 2015

Academic Editor: Zidong Wang

Copyright (C) 2015 Guisheng Jiang et al. This is an open access article distributed under the Creative Commons Attribution License, which permits unrestricted use, distribution, and reproduction in any medium, provided the original work is properly cited.

The algebraic connectivity of a graph is defined as the second smallest eigenvalue of the Laplacian matrix of the graph, which is a parameter to measure how well a graph is connected. In this paper, we present two unique graphs whose algebraic connectivity attain the minimum among all graphs whose complements are trees, but not stars, and among all graphs whose complements are unicyclic graphs, but not stars adding one edge, respectively.

\section{Introduction}

Let $G=(V(G), E(G))$ be a simple graph with vertex set $V(G)=\left\{v_{1}, v_{2}, \ldots, v_{n}\right\}$. The complement of $G$ is denoted by $G^{c}:=\left(V(G), E^{\prime}(G)\right)$, where $E^{\prime}(G)=\{x y: x, y \in V(G), x y \notin$ $E(G)\}$. The adjacency matrix of $G$ is defined to be a matrix $A(G)=\left[a_{i j}\right]$ of order $n$, where $a_{i j}=1$ if $v_{i}$ is adjacent to $v_{j}$, and $a_{i j}=0$ otherwise. The degree matrix of $G$ is denoted by $D(G)=\operatorname{diag}\left(d_{G}\left(v_{1}\right), d_{G}\left(v_{2}\right), \ldots, d_{G}\left(v_{n}\right)\right)$, where $d_{G}(v)$ denotes the degree of a vertex $v$ in the graph $G$. The matrix $Q(G)=D(G)+A(G)$ is the signless Laplacian matrix of $G$. The Laplacian matrix of $G$ is defined to be $L(G)=D(G)-A(G)$. Since $A(G), Q(G)$, and $L(G)$ are real and symmetric, their eigenvalues are real and can be arranged, respectively. We simply call the eigenvalues of $A(G)$ as eigenvalues of $G$, the largest eigenvalue of $A(G)$ as spectral radius of $G$, the eigenvalues of $Q(G)$ as the signless Laplacian eigenvalues of $G$, and the eigenvalues of $L(G)$ as the Laplacian eigenvalues of $G$. The second smallest Laplacian eigenvalue of $G$ is called the algebraic connectivity of $G$, denoted by $a(G)$. The eigenvectors corresponding to $a(G)$ are called Fiedler vectors of $G . a(G)$ is a good parameter to measure how well a graph is connected and plays an important role in control theory and communications, and so forth. In particular, it is related to the synchronization ability of complex network $[1,2]$. On the other hand it is related to the convergence speed in networks; one important topic is to increase $a(G)$ as much as possible. There are several techniques to enhance this metric; see [3].

When the structures of graphs are very complex, but the structures of their complements are simple, we naturally think whether we can study the graphs by studying their complements. Recently, there are some works about this subject. A connected graph $G$ with $n$ vertices and $m$ edges is called $k$-cyclic graph if $m=n-1+k$. Specially, if $k=$ $0,1,2$, or 3 , then $G$ is called a tree, unicyclic graph, bicyclic graph, or tricyclic graph, respectively. Let $\mathscr{T}_{n}$ be the set of trees on $n$ vertices, and let $\delta_{n}$ be the set of unicyclic graphs of order $n$. Denote by $K_{1, n-1}$ the star graph on $n$ vertices, and $K_{1, n-1}+e$ the graph obtained from $K_{1, n-1}$ by adding one edge $e$. Fan et al. [4] have got the unique minimizing graph whose least eigenvalue attains the minimum among all graphs $G$ for $G^{c} \in \mathscr{T}_{n} \backslash\left\{K_{1, n-1}\right\}$. Li and Wang [5] have researched the unique minimizing graph which whose signless Laplacian eigenvalue attains the minimum among all graphs $G$ for $G^{c} \in \mathscr{T}_{n} \backslash\left\{K_{1, n-1}\right\}$. Liu and Zhang [6] have studied the unique maximizing graph whose spectral radius attains the maximum among all graphs $G$ for $G^{c} \in \mathcal{S}_{n}$. Yin and Guo $[7,8]$ have characterized two maximizing graphs whose spectral radius attains the maximum among all the complements of bicyclic graphs of order $n$ and among all the complements of tricyclic graphs of order $n$, respectively. 
Yu and Fan $[9,10]$ have studied two unique minimizing graphs whose least eigenvalue attains the minimum among all graphs of order $n$ whose complements are connected and whose complements are connected and have no cut edges or cut vertices, that is, 2-edge connected or 2-vertex connected graphs, respectively.

We note that the least Laplacian eigenvalue of a graph is zero, and Fiedler [11] proves that a graph $G$ is connected if and only if $a(G)>0$. In fact, $K_{1, n-1}$ is the unique graph whose complement is not connected in $\mathscr{T}_{n}$, and $K_{1, n-1}+e$ is the unique graph whose complement is not connected in $\delta_{n}$. One may naturally ask what is the minimizing graph whose second smallest Laplacian eigenvalue (or the algebraic connectivity) attains the minimum among all graphs $G$ for $G^{c} \in \mathscr{T}_{n} \backslash\left\{K_{1, n-1}\right\}$ or for $G^{c} \in \mathcal{S}_{n} \backslash\left\{K_{1, n-1}+e\right\}$ ? In this paper, we study those questions and obtain two unique graphs which have the least algebraic connectivity among all graphs $G$ for $G^{c} \in \mathscr{T}_{n} \backslash\left\{K_{1, n-1}\right\}$ and for $G^{c} \in \mathcal{S}_{n} \backslash\left\{K_{1, n-1}+e\right\}$, in Sections 3 and 4 , respectively.

\section{Preliminaries}

We begin with some definitions. Given a graph $G$ of order $n$, a vector $X \in R^{n}$ is called to be defined on $G$, if there is a 1-1 map $\varphi$ from $V(G)$ to the entries of $X$, simply written $X_{u}=\varphi(u)$ for each $u \in V(G)$. If $X$ is an eigenvector of $L(G)$, then $X$ is defined on $G$ naturally; $X_{u}$ is the entry of $X$ corresponding to the vertex $u$. One can find that, for an arbitrary vector $X \in R^{n}$,

$$
X^{T} L(G) X=\sum_{u v \in E(G)}\left(X_{u}-X_{v}\right)^{2},
$$

and when $\mu$ is a Laplacian eigenvalue of $G$ corresponding to the eigenvector $X$ if and only if $X \neq \mathbf{0}$,

$$
\left(d_{G}(v)-\mu\right) X_{v}=\sum_{u \in N_{G}(v)} X_{u} \text {, for each vertex } v \in V(G),
$$

where $N_{G}(v)$ denotes the neighborhood of $v$ in the graph $G$. Equation (2) is called the Laplacian eigenvalue-equation for the graph $G$. In addition, by the well-known Courant-Fisher Theorem [11], for an arbitrary unit vector $X \in R^{n}$, when $X \neq$ $\mathbf{0}, X \perp \mathbf{1}$,

$$
a(G) \leq X^{T} L(G) X
$$

with equality if and only if $X$ is a Fiedler vector of $G$, where $\mathbf{0}$ is null vector and $\mathbf{1}$ is the vector such that each of its coordinates is equal to 1 .

Let $G^{c}$ be the complement of a graph $G$ of order $n$. It is easily seen that $L\left(G^{c}\right)=n \mathbf{I}-\mathbf{J}-L(G)$, where $\mathbf{J}$, I, respectively, denote the all-ones square matrix and the identity matrix both of suitable sizes. So for an arbitrary vector $X \in R^{n}$,

$$
X^{T} L\left(G^{c}\right) X=X^{T}(n \mathbf{I}-\mathbf{J}) X-X^{T} L(G) X .
$$

Lemma 1 (see [11]). Let $G$ be a simple graph. Then $a(G) \leq$ $\delta(G)$, where $\delta(G)=\min \left\{d_{G}(v), v \in V(G)\right\}$.

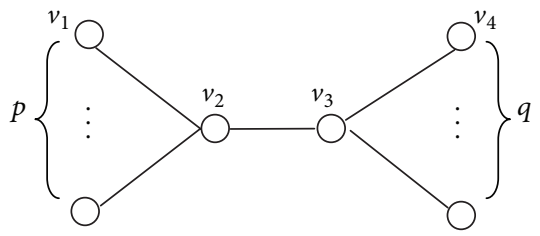

FIgURE 1: The graph $T(p, q)$.

Lemma 2. If $\left\{X_{i}, i=1,2, \ldots, n\right\}$ is a nonincreasing sequence, then, for any $1 \leq i, j \leq n,\left(X_{i}-X_{j}\right)^{2} \leq \max \left\{\left(X_{i}-X_{1}\right)^{2},\left(X_{i}-\right.\right.$ $\left.\left.X_{n}\right)^{2}\right\} \leq\left(X_{1}-X_{n}\right)^{2}$.

Proof. For any $1 \leq i, j \leq n$, if $X_{i}-X_{j} \geq 0$, by the monotone of $\left\{X_{i}, i=1,2, \ldots, n\right\}$, we have

$$
0 \leq X_{i}-X_{j} \leq X_{i}-X_{n} \leq X_{1}-X_{n}
$$

Then

$$
\left(X_{i}-X_{j}\right)^{2} \leq\left(X_{i}-X_{n}\right)^{2} \leq\left(X_{1}-X_{n}\right)^{2}
$$

Similarly, if $X_{i}-X_{j} \leq 0$, we have

$$
0 \geq X_{i}-X_{j} \geq X_{i}-X_{1} \geq X_{n}-X_{1}
$$

Then

$$
\left(X_{i}-X_{j}\right)^{2} \leq\left(X_{i}-X_{1}\right)^{2} \leq\left(X_{1}-X_{n}\right)^{2}
$$

The result follows.

\section{The least Algebraic Connectivity of the Complements of Trees}

Given a graph $G$ with $u, v \in V(G)$, let $d_{G}(u, v)$ be the distance between $u$ and $v$ in $G$. Denote by $K_{p, q}$ a complete bipartite graph whose bipartition has $p$ vertices and $q$ vertices, respectively. Denote by $T(p, q)$ the special tree, which is obtained from two disjoint stars $K_{1, p}(p \geq 1)$ and $K_{1, q}(q \geq 1)$ by joining the center of $K_{1, p}$ and $K_{1, q}$ by a path of length 1 ; see Figure 1.

Lemma 3. Given a positive integer $n(n \geq 6)$, for any positive integers $p, q$ with $p+q=n-2, p \geq q \geq 2$, one has

$$
a\left(T(p, q)^{c}\right)>a\left(T(p+1, q-1)^{c}\right) .
$$

Proof. Let $T(p, q)$ be the graph as depicted in Figure 1 with some vertices labeled. Let $X$ be a unit Fiedler vector of $T(p, q)^{c}$. By Lemma 1, we have $a\left(T(p, q)^{c}\right) \neq d_{T(p, q)^{c}}(v)+1$ for any vertex $v \in V\left(T(p, q)^{c}\right)$. Then by the Laplacian eigenequations (2), all vertices which are the pendant vertices of $v_{2}$ have the same values given by $X$, say $X_{1}$; all vertices which are the pendant vertices of $v_{3}$ have the same values given by $X$, say $X_{4}$. Write $X_{2}:=X_{v_{2}}, X_{3}:=X_{v_{3}}$. Now considering 
the Laplacian eigen-equations of $X$ for $T(p, q)^{c}$, and simply writing $a:=a\left(T(p, q)^{c}\right)$, we have

$$
\begin{gathered}
(p+q-a) X_{1}=(p-1) X_{1}+X_{3}+q X_{4}, \\
(q-a) X_{2}=q X_{4} \\
(p-a) X_{3}=p X_{1} \\
(p+q-a) X_{4}=p X_{1}+X_{2}+(q-1) X_{4} .
\end{gathered}
$$

Transform (10) into a matrix equation $(B-a \mathbf{I}) X^{\prime}=0$, where $X^{\prime}=\left(X_{1}, X_{2}, X_{3}, X_{4}\right)^{T}$ and

$$
B=\left[\begin{array}{cccc}
q+1 & 0 & -1 & -q \\
0 & q & 0 & -q \\
-p & 0 & p & 0 \\
-p & -1 & 0 & p+1
\end{array}\right] .
$$

Let $f(\mu ; p, q):=\operatorname{det}(\mu I-B)$; then we have

$$
\begin{aligned}
f(\mu ; p, q)= & \mu^{4}-2(p+q+1) \mu^{3} \\
& +\left(p^{2}+3 p q+2 p+q^{2}+2 q+1\right) \mu^{2} \\
& -p q(p+q+2) \mu .
\end{aligned}
$$

Because 0 and $a$ are roots of the polynomial $f(\mu ; p, q)$, then $a$ is the second smallest root of the polynomial $f(\mu ; p, q)$. Observe that

$$
\begin{aligned}
f & (\mu ; p+1, q-1)-f(\mu ; p, q) \\
& =\mu(q-p-1)(\mu-p-q-2) \\
& =\mu(q-p-1)(\mu-n) .
\end{aligned}
$$

By Lemma 1 , we have $a=a\left(T(p, q)^{c}\right)<n$. Then by $p \geq q$, $a>0$, and $f(a ; p, q)=0$, we have that

$$
f(a ; p+1, q-1)=a(q-p-1)(a-n)>0,
$$

which implies that

$$
a\left(T(p+1, q-1)^{c}\right)<a\left(T(p, q)^{c}\right) .
$$

The result now follows from the above discussion.

Corollary 4. Given a positive integer $n(n \geq 6)$, for any positive integers $p, q$ with $p+q=n-2, p \geq q \geq 2$, one has

$$
a\left(T(p, q)^{c}\right)>a\left(T(p+1, q-1)^{c}\right)>\cdots>a\left(T(n-3,1)^{c}\right) .
$$

Lemma 5. Given a positive integer $n(n \geq 4)$, for any tree $T \in$ $\mathcal{T}_{n} \backslash\left\{K_{1, n-1}\right\}$, there exist some integers $p, q$ with $1 \leq p, q \leq n-3$, $p+q=n-2$, such that

$$
a\left(T^{c}\right) \geq a\left(T(p, q)^{c}\right) .
$$

Proof. Let $X$ be a unit Fiedler vector of $T^{c}$; then $X \neq \mathbf{0}$ and $X \perp 1$. Thus we can get a sequence $\left\{X_{v_{i}}, i=1,2, \ldots, n\right\}$ such that

$$
X_{v_{1}} \geq X_{v_{2}} \geq \cdots \geq X_{v_{n}} .
$$

If $d_{T}\left(v_{1}, v_{n}\right)>1$, we can let the path $v_{1} T v_{n}=v_{1} u_{1} \cdots u_{2} v_{n}$, where $u_{1}=u_{2}$ when $d_{T}\left(v_{1}, v_{n}\right)=2$. Add the edge $v_{1} v_{n}$, and delete the edge $v_{1} u_{1}$ or $u_{2} v_{n}$ such that the result tree $T^{*}$ is not star. Then, by (1) and Lemma 2, we have

$$
\begin{aligned}
X^{T} L(T) X & =\sum_{v_{i} v_{j} \in E(T)}\left(X_{v_{i}}-X_{v_{j}}\right)^{2} \\
& \leq \sum_{v_{i} v_{j} \in E\left(T^{*}\right)}\left(X_{v_{i}}-X_{v_{j}}\right)^{2} \\
& =X^{T} L\left(T^{*}\right) X .
\end{aligned}
$$

If $T^{*} \not T(p, q)$ and there exists a pendant vertex $v$, whose neighbor $u$ is neither $v_{1}$ nor $v_{n}$, satisfying

$$
\left(X_{v}-X_{v_{1}}\right)^{2} \geq\left(X_{v}-X_{v_{n}}\right)^{2},
$$

then delete $u v$ and add $v v_{1}$; otherwise delete $u v$ and add $v v_{n}$. Repeat this rearranging until the resulting tree $T^{\prime} \cong T(p, q)$. By (1) and Lemma 2, we have

$$
\begin{aligned}
X^{T} L\left(T^{*}\right) X & =\sum_{v_{i} v_{j} \in E\left(T^{*}\right)}\left(X_{v_{i}}-X_{v_{j}}\right)^{2} \\
& \leq \sum_{v_{i} v_{j} \in E\left(T^{\prime}\right)}\left(X_{v_{i}}-X_{v_{j}}\right)^{2} \\
& =X^{T} L(T(p, q)) X .
\end{aligned}
$$

By (3), (4), (19), and (21), we have

$$
\begin{aligned}
a\left(T^{c}\right) & =X^{T} L\left(T^{c}\right) X \\
& =X^{T}(n \mathbf{I}-\mathbf{J}) X-X^{T} L(T) X \\
& \geq X^{T}(n \mathbf{I}-\mathbf{J}) X-X^{T} L(T(p, q)) X \\
& =X^{T} L\left(T(p, q)^{c}\right) X \\
& \geq a\left(T(p, q)^{c}\right) .
\end{aligned}
$$

The result follows.

By Corollary 4 and Lemma 5, we now obtain one main result of this paper.

Theorem 6. For $n \geq 4, T \in \mathscr{T}_{n} \backslash\left\{K_{1, n-1}\right\}$, one has

$$
a\left(T^{c}\right) \geq a\left(T(n-3,1)^{c}\right),
$$

with equality if and only if $T \cong T(n-3,1)$. 


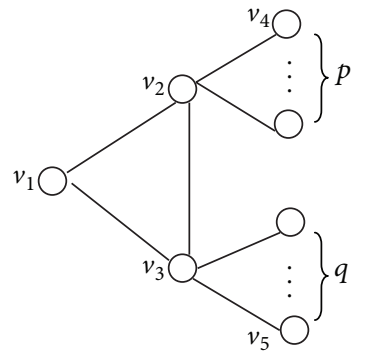

Figure 2: The graph $G_{1}(p, q)$.

\section{The Least Algebraic Connectivity of the Complements of Unicyclic Graphs}

Denote by $G_{1}(p, q)$ the special unicyclic graph, which is obtained from $C_{3}$ by identifying two vertices with the center of $K_{1, p}$ and the center of $K_{1, q}$, respectively; see Figure 2 .

Lemma 7. Given a positive integer $n(n \geq 7)$, for any positive integers $p, q$ with $p+q=n-3, p \geq q \geq 2$, we have

$$
a\left(G_{1}(p, q)^{c}\right)>a\left(G_{1}(p+1, q-1)^{c}\right) .
$$

Proof. Let $G_{1}(p, q)$ be the graph as depicted in Figure 2 with some vertices labeled. Let $X$ be a unit Fiedler vector of $G_{1}(p, q)^{c}$. By Lemma 1 , we have $a\left(G_{1}(p, q)^{c}\right) \neq d_{G_{1}(p, q)^{c}}(v)+1$ for any vertex $v \in V\left(G_{1}(p, q)^{c}\right)$. Then, by the Laplacian eigenequations (2), all vertices which are the pendant vertices of $v_{2}$ have the same values given by $X$, say $X_{4}$; all vertices which are the pendant vertices of $v_{3}$ have the same values given by $X$, say $X_{5}$. Write $X_{i}:=X_{v_{i}} i=1,2,3$. Now considering the Laplacian eigen-equations of $X$ for $G_{1}(p, q)^{c}$, and simply writing $a:=a\left(G_{1}(p, q)^{c}\right)$, we have

$$
\begin{gathered}
(p+q-a) X_{1}=p X_{4}+q X_{5}, \\
(q-a) X_{2}=q X_{5} \\
(p-a) X_{3}=p X_{4} \\
(p+q+1-a) X_{4}=X_{1}+X_{3}+(p-1) X_{4}+q X_{5} \\
(p+q+1-a) X_{5}=X_{1}+X_{2}+p X_{4}+(q-1) X_{5} .
\end{gathered}
$$

Transform (25) into a matrix equation $(B-a \mathbf{I}) X^{\prime}=0$, where $X^{\prime}=\left(X_{1}, X_{2}, X_{3}, X_{4}, X_{5}\right)^{T}$ and

$$
B=\left[\begin{array}{ccccc}
p+q & 0 & 0 & -p & -q \\
0 & q & 0 & 0 & -q \\
0 & 0 & p & -p & 0 \\
-1 & 0 & -1 & q+2 & -q \\
-1 & -1 & 0 & -p & p+2
\end{array}\right] .
$$

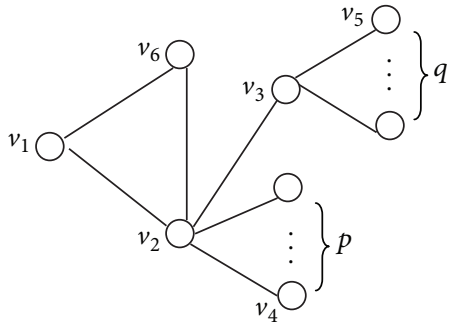

Figure 3: The graph $G_{2}(p, q)$.

Let $f_{1}(\mu ; p, q):=\operatorname{det}(\mu I-B)$; then we have

$$
\begin{aligned}
f_{1}(\mu ; p, q)= & \mu^{5}-(3 p+3 q+4) \mu^{4} \\
& +\left(3 p^{2}+7 p q+8 p+3 q^{2}+8 q+4\right) \mu^{3} \\
& -(p+q+2)\left(p^{2}+4 p q+2 p+q^{2}+2 q\right) \mu^{2} \\
& +p q(p+q+1)(p+q+3) \mu .
\end{aligned}
$$

Because 0 and $a$ are roots of the polynomial $f_{1}(\mu ; p, q)$, then $a$ is the second smallest root of the polynomial $f_{1}(\mu ; p, q)$. Observe that

$$
\begin{aligned}
& f_{1}(\mu ; p+1, q-1)-f_{1}(\mu ; p, q) \\
& \quad=-\mu(p-q+1)(\mu-p-q-1)(\mu-p-q-3) .
\end{aligned}
$$

By Lemma 1, we have $a=a\left(G_{1}(p, q)^{c}\right) \leq q<p+q+1$. Then, by $p \geq q \geq 2, a>0$, and $f_{1}(a ; p, q)=0$, we have

$$
\begin{aligned}
& f_{1}(a ; p+1, q-1) \\
& \quad=-a(p-q+1)(a-p-q-1)(a-p-q-3)<0,
\end{aligned}
$$

which implies that

$$
a\left(G_{1}(p+1, q-1)^{c}\right)<a\left(G_{1}(p, q)^{c}\right)
$$

The result now follows from the above discussion.

Corollary 8. Given a positive integer $n(n \geq 7)$, for any positive integers $p, q$ with $p+q=n-3, p \geq q \geq 2$, one has

$$
\begin{aligned}
a\left(G_{1}(p, q)^{c}\right) & >a\left(G_{1}(p+1, q-1)^{c}\right) \\
& >\cdots>a\left(G_{1}(n-4,1)^{c}\right) .
\end{aligned}
$$

Denote by $G_{2}(p, q)$ the special unicyclic graph, which is obtained from $C_{3}$ by identifying one vertex with the center of $K_{1, p}$ and the pendent vertex of $K_{1, q+1}$; see Figure 3 . 
Lemma 9. Given a positive integer $n(n \geq 8)$, for any positive integers $p, q$ with $p+q=n-4$, one has

$$
\begin{gathered}
a\left(G_{2}(p, q)^{c}\right)>a\left(G_{2}(p+1, q-1)^{c}\right), \quad \text { if } p+3>q \geq 2 \\
a\left(G_{2}(p, q)^{c}\right)=a\left(G_{2}(p+1, q-1)^{c}\right), \quad \text { if } p+3=q \\
a\left(G_{2}(p, q)^{c}\right)<a\left(G_{2}(p+1, q-1)^{c}\right), \quad \text { if } p+3<q .
\end{gathered}
$$

Proof. Let $G_{2}(p, q)$ be the graph as depicted in Figure 3 with some vertices labeled. Let $X$ be a unit Fiedler vector of $G_{2}(p, q)^{c}$. By Lemma 1, we have $a\left(G_{2}(p, q)^{c}\right) \neq d_{G_{2}(p, q)^{c}}(v)+$ 1 for any vertex $v \in V\left(G_{2}(p, q)^{c}\right)$ and $a\left(G_{2}(p, q)^{c}\right) \neq$ $d_{G_{2}(p, q)^{c}}\left(v_{1}\right)$. Write $X_{i}:=X_{v_{i}}, i=1,2,3,4,5,6$. Then by the Laplacian eigen-equations (2), $X_{1}=X_{6}$, all vertices which are the pendant vertices of $v_{2}$ have the same values given by $X$, namely, $X_{4}$; all vertices which are the pendant vertices of $v_{3}$ have the same values given by $X$, namely, $X_{5}$. Now considering the Laplacian eigen-equations of $X$ for $G_{2}(p, q)^{c}$, and simply writing $a:=a\left(G_{2}(p, q)^{c}\right)$, we have

$$
\begin{gathered}
(p+q+1-a) X_{1}=X_{3}+p X_{4}+q X_{5}, \\
(q-a) X_{2}=q X_{5}, \\
(p+2-a) X_{3}=2 X_{1}+p X_{4}, \\
(p+q+2-a) X_{4}=2 X_{1}+X_{3}+(p-1) X_{4}+q X_{5}, \\
(p+q+2-a) X_{5}=2 X_{1}+X_{2}+p X_{4}+(q-1) X_{5} .
\end{gathered}
$$

Transform (33) into a matrix equation $(B-a \mathbf{I}) X^{\prime}=0$, where $X^{\prime}=\left(X_{1}, X_{2}, X_{3}, X_{4}, X_{5}\right)^{T}$ and

$$
B=\left[\begin{array}{ccccc}
p+q+1 & 0 & -1 & -p & -q \\
0 & q & 0 & 0 & -q \\
-2 & 0 & p+2 & -p & 0 \\
-2 & 0 & -1 & q+3 & -q \\
-2 & -1 & 0 & -p & p+3
\end{array}\right] .
$$

Let $f_{2}(\mu ; p, q):=\operatorname{det}(\mu I-B)$; then we have

$$
\begin{aligned}
f_{2}(\mu ; p, q)= & \mu^{5}-(3 p+3 q+9) \mu^{4} \\
& +\left(3 p^{2}+7 p q+18 p+3 q^{2}+20 q+27\right) \mu^{3} \\
& -\left(p^{3}+5 p^{2} q+9 p^{2}+5 p q^{2}+29 p q+27 p\right. \\
& \left.+q^{3}+13 q^{2}+41 q+27\right) \mu^{2} \\
& +q(p+2)(p+q+3)(p+q+4) \mu .
\end{aligned}
$$

Because 0 and $a$ are roots of the polynomial $f_{2}(\mu ; p, q)$, then $a$ is the second smallest root of the polynomial $f_{2}(\mu ; p, q)$. Observe that

$$
\begin{aligned}
& f_{2}(\mu ; p+1, q-1)-f_{2}(\mu ; p, q) \\
& \quad=-\mu(p-q+3)(p+q-\mu+3)(p+q-\mu+4) .
\end{aligned}
$$

Because $f_{2}(a ; p, q)=0$, we have

$$
\begin{aligned}
& f_{2}(a ; p+1, q-1) \\
& \quad=-a(p-q+3)(p+q-a+3)(p+q-a+4) .
\end{aligned}
$$

By Lemma 1, we note that $0<a=a\left(G_{2}(p, q)^{c}\right) \leq p+q+2$.

If $p+3>q \geq 2$, we have $f_{2}(a ; p+1, q-1)<0$, which implies that

$$
a\left(G_{2}(p+1, q-1)^{c}\right)<a\left(G_{2}(p, q)^{c}\right) .
$$
that

If $p+3=q$, we have $f_{2}(a ; p+1, q-1)=0$, which implies

$$
a\left(G_{2}(p+1, q-1)^{c}\right)=a\left(G_{2}(p, q)^{c}\right) .
$$

If $p+3<q$, we have

$$
f_{2}(a ; p+1, q-1)>0
$$

and $0<a=a\left(G_{2}(p, q)^{c}\right) \leq p+2$ by Lemma 1 .

Let

$$
f_{2}(\mu ; p, q)=\mu(p+q-\mu+3) g(\mu)
$$

Then

$$
\begin{aligned}
g(\mu)= & -\mu^{3}+(2 p+2 q+6) \mu^{2} \\
& -\left(p^{2}+3 p q+6 p+q^{2}+8 q+9\right) \mu \\
& +p^{2} q+p q^{2}+6 p q+2 q^{2}+8 q,
\end{aligned}
$$

and $a$ is the least root of the polynomial $g(\mu)$ by $0<a \leq p+2$. We calculate that

$$
\begin{aligned}
g^{\prime}(\mu)= & -3 \mu^{2}+(4 p+4 q+12) \mu-p^{2}-3 p q \\
& -6 p-q^{2}-8 q-9, \\
& g^{\prime \prime}(\mu)=-6 \mu+4 p+4 q+12 .
\end{aligned}
$$

Thus when $0<\mu \leq p+2, p+3<q, g^{\prime \prime}(\mu) \geq g^{\prime \prime}(p+$ $2)=4 q-2 p>0$, which implies that $g^{\prime}(\mu)$ is monotonously increasing for $\mu$ when $0<\mu \leq p+2, p+3<q$.

So, when $0<\mu \leq p+2, p+3<q, g^{\prime}(\mu) \leq g^{\prime}(p+2)=$ $-q^{2}+p q+2 p+3<-q-3<0$, which implies that $g(\mu)$ is monotonously decreasing for $\mu$ when $0<\mu \leq p+2, p+3<q$.

Then, if $p+3<q$, by $f_{2}(a ; p+1, q-1)>0$, we get

$$
a\left(G_{2}(p+1, q-1)^{c}\right)>a\left(G_{2}(p, q)^{c}\right) .
$$

The result now follows from the above discussion.

Lemma 10. Given a positive integer $n(n \geq 8)$, for any positive integers $p, q$ with $p+q=n-4, q \geq 1$, one has

$$
a\left(G_{2}(p, q)^{c}\right) \geq a\left(G_{2}(n-5,1)^{c}\right),
$$

with equality if and only if $G_{2}(p, q)=G_{2}(n-5,1)$. 


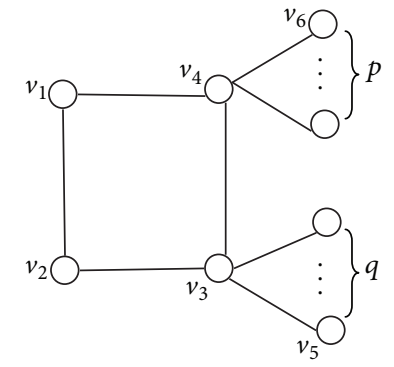

Figure 4: The graph $G_{3}(p, q)$.

Proof. From the proof of Lemma 9, we note that $a_{1}^{\prime}:=$ $a\left(G_{2}(n-5,1)^{c}\right), a_{2}^{\prime}:=a\left(G_{2}(0, n-4)^{c}\right)$ are the second smallest roots of the following two polynomials, respectively:

$$
\begin{aligned}
& f_{2}(n-5,1) \\
& \quad \begin{aligned}
& \\
=\mu(\mu-n+1) & \left(\mu^{3}+(2-2 n) \mu^{2}\right. \\
& \left.+\left(n^{2}-n-2\right) \mu+3 n-n^{2}\right), \\
f_{2}(0, n-4) & \\
=\mu(\mu-n+1) & \left(\mu^{3}+(2-2 n) \mu^{2}\right. \\
& \left.+\left(n^{2}-7\right) \mu+8 n-2 n^{2}\right) .
\end{aligned}
\end{aligned}
$$

By Lemma 1 and $n \geq 8$, we have $0<a_{1}^{\prime}, a_{2}^{\prime} \leq 2<$ $n-1$. Thus, $a_{1}^{\prime}, a_{2}^{\prime}$ are the least roots of the following two polynomials, respectively:

$$
\begin{gathered}
h(\mu)=\mu^{3}+(2-2 n) \mu^{2}+\left(n^{2}-n-2\right) \mu+3 n-n^{2}, \\
k(\mu)=\mu^{3}+(2-2 n) \mu^{2}+\left(n^{2}-7\right) \mu+8 n-2 n^{2} .
\end{gathered}
$$

We observe that

$$
h(\mu)-k(\mu)=(n-\mu)(n-5)>0,
$$

for $0<\mu \leq 2$ and $n \geq 8$.

Because $k\left(a_{2}^{\prime}\right)=0, n \geq 8$, and $0<a_{2}^{\prime} \leq 2$, we have

$$
h\left(a_{2}^{\prime}\right)>0,
$$

which imply that $a_{1}^{\prime}<a_{2}^{\prime}$; namely,

$$
a\left(G_{2}(n-5,1)^{c}\right)<a\left(G_{2}(0, n-4)^{c}\right) .
$$

By Lemma 9, the result follows.

Denote by $G_{3}(p, q)$ the special unicyclic graph, which is obtained from $C_{4}$ by identifying two adjacent vertices with the center of $K_{1, p}$ and the center of $K_{1, q}$, respectively; see Figure 4.

Lemma 11. Given a positive integer $n(n \geq 6)$, for any positive integers $p, q$ with $p+q=n-4, p \geq q \geq 1$, one has

$$
a\left(G_{3}(p, q)^{c}\right)>a\left(G_{3}(p+1, q-1)^{c}\right) .
$$

Proof. Let $G_{3}(p, q)$ be the graph as depicted in Figure 4 with some vertices labeled. Let $X$ be a unit Fiedler vector of $G_{3}(p, q)^{c}$. By Lemma 1, we have $a\left(G_{3}(p, q)^{c}\right) \neq d_{G_{3}(p, q)^{c}}(v)+1$ for any vertex $v \in V\left(G_{3}(p, q)^{c}\right)$. Write $X_{i}:=X_{v_{i}}, i=$ $1,2,3,4,5,6$. Then by the Laplacian eigen-equations (2), all vertices which are the pendant vertices of $v_{3}$ have the same values given by $X$, namely, $X_{5}$; all vertices which are the pendant vertices of $v_{4}$ have the same values given by $X$, namely, $X_{6}$. Now considering the Laplacian eigen-equations of $X$ for $G_{3}(p, q)^{c}$, and simply writing $a:=a\left(G_{3}(p, q)^{c}\right)$, we have

$$
\begin{gathered}
(p+q+1-a) X_{1}=X_{3}+q X_{5}+p X_{6}, \\
(p+q+1-a) X_{2}=X_{4}+q X_{5}+p X_{6}, \\
(p+1-a) X_{3}=X_{1}+p X_{6}, \\
(q+1-a) X_{4}=X_{2}+q X_{5}, \\
(p+q+2-a) X_{5}=X_{1}+X_{2}+X_{4}+(q-1) X_{5}+p X_{6}, \\
(p+q+2-a) X_{6}=X_{1}+X_{2}+X_{3}+q X_{5}+(p-1) X_{6} .
\end{gathered}
$$

Transform (52) into a matrix equation $(B-a \mathbf{I}) X^{\prime}=0$, where $X^{\prime}=\left(X_{1}, X_{2}, X_{3}, X_{4}, X_{5}, X_{6}\right)^{T}$ and

$$
B=\left[\begin{array}{cccccc}
p+q+1 & 0 & -1 & 0 & -q & -p \\
0 & p+q+1 & 0 & -1 & -q & -p \\
-1 & 0 & p+1 & 0 & 0 & -p \\
0 & -1 & 0 & q+1 & -q & 0 \\
-1 & -1 & 0 & -1 & p+3 & -p \\
-1 & -1 & -1 & 0 & -q & q+3
\end{array}\right] .
$$

Let $f_{3}(\mu ; p, q):=\operatorname{det}(\mu I-B)$; then we have

$$
\begin{aligned}
f_{3}(\mu ; p, q) & \\
= & \mu^{6}-(4 p+4 q+10) \mu^{5} \\
+ & \left(6 p^{2}+13 p q+31 p+6 q^{2}+31 q+37\right) \mu^{4} \\
- & \left(4 p^{3}+15 p^{2} q+33 p^{2}+15 p q^{2}+74 p q\right. \\
& \left.+83 p+4 q^{3}+33 q^{2}+83 q+60\right) \mu^{3} \\
+ & \left(p^{4}+7 p^{3} q+13 p^{3}+12 p^{2} q^{2}+55 p^{2} q\right. \\
& +55 p^{2}+7 p q^{3}+55 p q^{2}+129 p q+86 p+q^{4} \\
& \left.+13 q^{3}+55 q^{2}+86 q+36\right) \mu^{2} \\
- & (p+q+3)(p+q+4) \\
& \left(p^{2} q+p^{2}+p q^{2}+3 p q+2 p+q^{2}+2 q\right) \mu .
\end{aligned}
$$


Because 0 and $a$ are roots of the polynomial $f_{3}(\mu ; p, q)$, then $a$ is the second smallest root of the polynomial $f_{3}(\mu$; $p, q)$. Observe that

$$
\begin{aligned}
f_{3} & (\mu ; p+1, q-1)-f_{3}(\mu ; p, q) \\
= & -\mu(p-q+1)(\mu-p-q-1)(\mu-p-q-4) \\
& \cdot(\mu-p-q-3) .
\end{aligned}
$$

By Lemma 1 , we have $a=a\left(G_{3}(p, q)^{c}\right) \leq q+1$. Then by $p \geq q \geq 1, a>0$, and $f_{3}(a ; p, q)=0$, we have

$$
\begin{aligned}
f_{3}(a ; p+1, q-1) \\
=-a(p-q+1)(a-p-q-1)(a-p-q-4) \\
\quad \cdot(a-p-q-3)>0,
\end{aligned}
$$

which implies that

$$
a\left(G_{3}(p+1, q-1)^{c}\right)<a\left(G_{3}(p, q)^{c}\right) .
$$

The result now follows from the above discussion.

Corollary 12. Given a positive integer $n(n \geq 6)$, for any positive integers $p, q$ with $p+q=n-4, p \geq q \geq 1$, one has

$$
\begin{aligned}
a\left(G_{3}(p, q)^{c}\right) & >a\left(G_{3}(p+1, q-1)^{c}\right) \\
& >\cdots>a\left(G_{3}(n-4,0)^{c}\right) .
\end{aligned}
$$

Lemma 13. Given a positive integer $n(n \geq 6)$, for any unicyclic graph $G \in \mathcal{S}_{n} \backslash\left\{K_{1, n-1}+e\right\}$, there exist some integers $p, q$ with $p, q \geq 1$, such that

$$
a\left(G^{c}\right) \geq a\left(G_{i}(p, q)^{c}\right), \quad i=1 \text { or } 2 \text { or } 3 .
$$

Proof. Let $G$ be obtained from $l$-cycle $C_{l}$ (the cycle which has length $l$ ) by attaching some trees to it, and let $X$ be a unit Fiedler vector of $G^{c}$. Then $X \neq \mathbf{0}$ and $X \perp \mathbf{1}$. Thus we can get a sequence $\left\{X_{v_{n}}\right\}$ such that

$$
X_{v_{1}} \geq X_{v_{2}} \geq \cdots \geq X_{v_{n}} .
$$

If $d_{G}\left(v_{1}, v_{n}\right)>1$, we can let the path $v_{1} G v_{n}=v_{1} u_{1} \cdots u_{2} v_{n}$, where $u_{1}=u_{2}$ when $d_{G}\left(v_{1}, v_{n}\right)=2$. Add the edge $v_{1} v_{n}$, and delete the edge $v_{1} u_{1}$ or $u_{2} v_{n}$ such that the result unicyclic graph $G_{*}$ is not $K_{1, n-1}+e$. Then, by (1) and Lemma 2, we have

$$
\begin{aligned}
X^{T} L(G) X & =\sum_{v_{i} v_{j} \in E(G)}\left(X_{v_{i}}-X_{v_{j}}\right)^{2} \\
& \leq \sum_{v_{i} v_{j} \in E\left(G_{*}\right)}\left(X_{v_{i}}-X_{v_{j}}\right)^{2}=X^{T} L\left(G_{*}\right) X,
\end{aligned}
$$

where $G_{*}$ is obtained from $C_{l^{\prime}}$ by attaching some trees to it and $d_{G_{x}}\left(v_{1}, v_{n}\right)=1$.

If $G_{*} \notin\left\{G_{1}(p, q), G_{2}(p, q), G_{3}(p, q)\right\}$, we will discuss $G_{*}$ in three cases in the following.

Case 1. Both $v_{1}$ and $v_{n}$ are on the cycle $C_{l^{\prime}}$.
If $l^{\prime} \geq 5$, we can let the cycle $C_{l^{\prime}}=v_{1} v_{n} u_{1} u_{2} u_{3} \cdots v_{1}$. In this time, if $\left(X_{u_{2}}-X_{v_{1}}\right)^{2} \geq\left(X_{u_{2}}-X_{v_{n}}\right)^{2}$, we delete the edge $u_{2} u_{3}$ and add the edge $v_{1} u_{2}$; otherwise, we delete the edge $u_{2} u_{3}$ and add the edge $u_{2} v_{n}$. Then the result unicyclic graph $G_{* *}$ is obtained from $C_{3}\left(v_{n}\right.$ is on the cycle) or $C_{4}$ (both $v_{1}$ and $v_{n}$ are on the cycle) by attaching some trees to it.

Case 2. One of $v_{1}, v_{n}$ is on the cycle $C_{l^{\prime}}$.

We may let $v_{1}$ be on the cycle $C_{l^{\prime}}$, and it has same discussion when $v_{n}$ is on the cycle $C_{l^{\prime}}$. If $l^{\prime} \geq 4$, we can let the cycle $C_{l^{\prime}}=v_{1} u_{1} u_{2} u_{3} \cdots v_{1}$. In this time, if $\left(X_{v_{1}}-X_{u_{2}}\right)^{2} \geq$ $\left(X_{u_{2}}-X_{v_{n}}\right)^{2}$, we delete the edge $u_{2} u_{3}$ and add the edge $v_{1} u_{2}$; otherwise, we delete the edge $u_{2} u_{3}$ and add the edge $u_{2} v_{n}$. Then the result unicyclic graph $G_{* *}$ is obtained from $C_{3}$ (one of $v_{1}, v_{n}$ is on the cycle) or $C_{4}$ (both $v_{1}$ and $v_{n}$ are on the cycle) by attaching some trees to it.

Case 3. Neither $v_{1}$ nor $v_{n}$ is on the cycle $C_{l^{\prime}}$.

We can let the cycle $C_{l^{\prime}}=u_{1} u_{2} u_{3} \cdots u_{1}$, and neither $v_{1}$ nor $v_{n}$ is on the tree which is attached to $u_{2}$. In this time, if $\left(X_{v_{1}}-X_{u_{2}}\right)^{2} \geq\left(X_{u_{2}}-X_{v_{n}}\right)^{2}$, we delete the edge $u_{2} u_{3}$ and add the edge $v_{1} u_{2}$; otherwise, we delete the edge $u_{2} u_{3}$ and add the edge $u_{2} v_{n}$. Then the result unicyclic graph $G_{* *}$ is in Case 1 or Case 2.

Then, by (1) and Lemma 2, we have

$$
\begin{aligned}
X^{T} L\left(G_{*}\right) X & =\sum_{v_{i} v_{j} \in E\left(G_{*}\right)}\left(X_{v_{i}}-X_{v_{j}}\right)^{2} \\
& \leq \sum_{v_{i} v_{j} \in E\left(G_{* *}\right)}\left(X_{v_{i}}-X_{v_{j}}\right)^{2}=X^{T} L\left(G_{* *}\right) X,
\end{aligned}
$$

where $d_{G_{* *}}\left(v_{1}, v_{n}\right)=1$ and $G_{* *}$ is obtained from $C_{3}$ by attaching some trees to it, where at least one of $v_{1}, v_{n}$ is on the cycle, or $G_{* *}$ is obtained from $C_{4}$ by attaching some trees to it, where both $v_{1}$ and $v_{n}$ are on the cycle.

If $G_{* * *} \notin\left\{G_{1}(p, q), G_{2}(p, q), G_{3}(p, q)\right\}$ and there exists a pendant vertex $v$, whose neighbor $u$ is neither $v_{1}$ nor $v_{n}$, satisfying

$$
\left(X_{v}-X_{v_{1}}\right)^{2} \geq\left(X_{v}-X_{v_{n}}\right)^{2}
$$

then delete $u v$ and add $v v_{1}$; otherwise delete $u v$ and add $v v_{n}$. Repeat this rearranging until the result graph $G_{* * *} \in$ $\left\{G_{1}(p, q), G_{2}(p, q), G_{3}(p, q)\right\}$.

Then by (1) and Lemma 2 , we have

$$
\begin{aligned}
X^{T} L\left(G_{* *}\right) X & =\sum_{v_{i} v_{j} \in E\left(G_{* *}\right)}\left(X_{v_{i}}-X_{v_{j}}\right)^{2} \\
& \leq \sum_{v_{i} v_{j} \in E\left(G_{* * *}\right)}\left(X_{v_{i}}-X_{v_{j}}\right)^{2}=X^{T} L\left(G_{* * *}\right) X .
\end{aligned}
$$


According to (3), (4), (61), (62), and (64), we have

$$
\begin{aligned}
a\left(G^{c}\right) & =X^{T} L\left(G^{c}\right) X \\
& =X^{T}(n \mathbf{I}-\mathbf{J}) X-X^{T} L(G) X \\
& \geq X^{T}(n \mathbf{I}-\mathbf{J}) X-X^{T} L\left(G_{*}\right) X \\
& \geq X^{T}(n \mathbf{I}-\mathbf{J}) X-X^{T} L\left(G_{* *}\right) X \\
& \geq X^{T}(n \mathbf{I}-\mathbf{J}) X-X^{T} L\left(G_{* * *}\right) X \\
& =X^{T} L\left(G_{* * *}^{c}\right) X \\
& \geq a\left(G_{* * *}^{c}\right) .
\end{aligned}
$$

The result follows.

Theorem 14. For $n \geq 8, G \in \mathcal{S}_{n} \backslash\left\{K_{1, n-1}+e\right\}$, one has

$$
a\left(G^{c}\right) \geq a\left(G_{3}(n-4,0)^{c}\right),
$$

with equality if and only if $G \cong G_{3}(n-4,0)$.

Proof. From the proofs of Lemmas 7, 9, and 11, we note that $a_{1}:=a\left(G_{1}(n-4,1)^{c}\right), a_{2}:=a\left(G_{2}(n-5,1)^{c}\right)$, and $a_{3}:=$ $a\left(G_{3}(n-4,0)^{c}\right)$ are the second smallest roots of the following three polynomials, respectively:

$$
\begin{aligned}
& f_{1}(\mu ; n-4,1) \\
& =\mu^{5}+(5-3 n) \mu^{4}+\left(3 n^{2}-9 n+3\right) \mu^{3} \\
& +\left(-n^{3}+3 n^{2}+3 n-5\right) \mu^{2}+\left(n^{3}-6 n^{2}+8 n\right) \mu \\
& =\mu\left(\mu^{4}-(3 n-5) \mu^{3}+\left(3 n^{2}-9 n+3\right) \mu^{2}\right. \\
& \left.-\left(n^{3}-3 n^{2}-3 n+5\right) \mu+n^{3}-6 n^{2}+8 n\right), \\
& f_{2}(\mu ; n-5,1) \\
& =\mu^{5}+(3-3 n) \mu^{4}+\left(3 n^{2}-5 n\right) \mu^{3} \\
& +\left(-n^{3}+n^{2}+4 n-2\right) \mu^{2}+n(n-1)(n-3) \mu \\
& =\mu(\mu-n+1) \\
& \cdot\left(\mu^{3}+(2-2 n) \mu^{2}+\left(n^{2}-n-2\right) \mu+3 n-n^{2}\right), \\
& f_{3}(\mu ; n-4,0) \\
& =\mu^{6}+(6-4 n) \mu^{5}+\left(6 n^{2}-17 n+9\right) \mu^{4} \\
& +\left(-4 n^{3}+15 n^{2}-11 n\right) \mu^{3} \\
& +\left(n^{4}-3 n^{3}-5 n^{2}+14 n-4\right) \mu^{2} \\
& +\left(-n^{4}+7 n^{3}-14 n^{2}+8 n\right) \mu \\
& =\mu(\mu-n+1)(\mu-n+2) \\
& \cdot\left(\mu^{3}+(3-2 n) \mu^{2}+\left(n^{2}-2 n-2\right) \mu+4 n-n^{2}\right) \text {. }
\end{aligned}
$$

By Lemma 1 and $n \geq 8$, we have $0<a_{1}, a_{2}, a_{3} \leq 1<$ $n-2$. Thus, $a_{1}, a_{2}, a_{3}$ are the least roots of the following three polynomials, respectively:

$$
\begin{gathered}
g_{1}(\mu)=\mu^{4}-(3 n-5) \mu^{3}+\left(3 n^{2}-9 n+3\right) \mu^{2} \\
-\left(n^{3}-3 n^{2}-3 n+5\right) \mu+n^{3}-6 n^{2}+8 n, \\
g_{2}(\mu)=\mu^{3}+(2-2 n) \mu^{2}+\left(n^{2}-n-2\right) \mu+3 n-n^{2}, \\
g_{3}(\mu)=\mu^{3}+(3-2 n) \mu^{2}+\left(n^{2}-2 n-2\right) \mu+4 n-n^{2} .
\end{gathered}
$$

Claim 1. Consider $a\left(G_{3}(n-4,0)^{c}\right)<a\left(G_{1}(n-4,1)^{c}\right)$.

We observe that

$$
\begin{aligned}
& f_{1}(\mu ; n-4,1)-\mu(\mu-n+2) g_{3}(\mu) \\
& \quad=\mu^{2}(n-\mu-1)>0,
\end{aligned}
$$

for $0<\mu \leq 1$ and $n \geq 8$.

Because $f_{1}\left(a_{1} ; n-4,1\right)=0, n \geq 8$, and $0<a_{1} \leq 1$, we have

$$
g_{3}\left(a_{1}\right)>0
$$

which imply that $a_{3}<a_{1}$; namely,

$$
a\left(G_{3}(n-4,0)^{c}\right)<a\left(G_{1}(n-4,1)^{c}\right) .
$$

Claim 2. $a\left(G_{3}(n-4,0)^{c}\right)<a\left(G_{2}(n-5,1)^{c}\right)$.

We observe that

$$
g_{2}(\mu)-g_{3}(\mu)=-\mu^{2}+n \mu-n<0,
$$

for $0<\mu \leq 1$.

Because $0<a_{3} \leq 1$ and $g_{3}\left(a_{3}\right)=0$, we have $g_{2}\left(a_{3}\right)<0$.

We note that

$$
\begin{aligned}
g_{2}^{\prime}(\mu) & =3 \mu^{2}+2(2-2 n) \mu+\left(n^{2}-n-2\right) \\
& >2(2-2 n)+\left(n^{2}-n-2\right) \\
& =n^{2}-5 n+2>0
\end{aligned}
$$

for $n \geq 8$ and $0<\mu \leq 1$.

Then $g_{2}(\mu)$ is monotonously increasing for $u$ when $n \geq 8$ and $0<\mu \leq 1$.

Thus by $g_{2}\left(a_{3}\right)<0, g_{2}\left(a_{2}\right)=0$, and $0<a_{2}, a_{3} \leq 1$, we gain $a_{3}<a_{2}$; that is,

$$
a\left(G_{3}(n-4,0)^{c}\right)<a\left(G_{2}(n-5,1)^{c}\right) .
$$

According to Corollary 8, Lemma 10, Corollary 12, Lemma 13, Claim 1, and Claim 2, the result follows.

\section{Conflict of Interests}

The authors declare that there is no conflict of interests regarding the publication of this paper. 


\section{Acknowledgments}

This work is jointly supported by the National Natural Science Foundation of China under Grant nos. 11071001 and 11071002, the Natural Science Foundation of Anhui Province of China under Grant no. 11040606M14, and the Natural Science Foundation of Department of Education of Anhui Province of China under Grant nos. KJ2013A196 and KJ2015ZD27.

\section{References}

[1] R. Olfati-Saber, J. A. Fax, and R. M. Murray, "Consensus and cooperation in networked multi-agent systems," Proceedings of the IEEE, vol. 95, no. 1, pp. 215-233, 2007.

[2] Y. Tang, H. Gao, and J. Kurths, "Distributed robust synchronization of dynamical networks with stochastic coupling," IEEE Transactions on Circuits and Systems I: Regular Papers, vol. 61, no. 5, pp. 1508-1519, 2014.

[3] Y. Tang, Z. Wang, H. Gao, S. Swift, and J. Kurths, "A constrained evolutionary computation method for detecting controlling regions of cortical networks," IEEE/ACM Transactions on Computational Biology and Bioinformatics, vol. 9, no. 6, pp. 15691581, 2012.

[4] Y.-Z. Fan, F.-F. Zhang, and Y. Wang, "The least eigenvalue of the complements of trees," Linear Algebra and Its Applications, vol. 435, no. 9, pp. 2150-2155, 2011.

[5] S. C. Li and S. J. Wang, "The least eigenvalue of the signless Laplacian of the complements of trees," Linear Algebra and its Applications, vol. 436, no. 7, pp. 2398-2405, 2012.

[6] J. Liu and Z. Zhang, "Spectral radius of the complement of unicyclic graphs," Journal of East China Normal University (Natural Science), no. 5, pp. 14-19, 2010.

[7] L.-W. Yin and S.-G. Guo, "Spectral radius of complement of bicyclic graphs," Journal of Yancheng Teachers University (Natural Science), vol. 26, no. 2, pp. 17-21, 2008.

[8] L.-W. Yin and S.-G. Guo, "Spectral radius of complement of tricyclic graphs with n vertices," Applied Mathematics-Series $A$, vol. 262, pp. 223-229, 2011.

[9] G. D. Yu and Y. Z. Fan, "The least eigenvalue of graphs," Journal of Mathematical Research with Applications, vol. 32, no. 6, pp. 659-665, 2012.

[10] G.-D. Yu and Y.-Z. Fan, "The least eigenvalue of graphs whose complements are 2-vertex or 2-edge connected," Operations Research Transactions, vol. 17, no. 2, pp. 81-88, 2013.

[11] M. Fiedler, "Algebraic connectivity of graphs," Czechoslovak Mathematical Journal, vol. 23(98), pp. 298-305, 1973. 


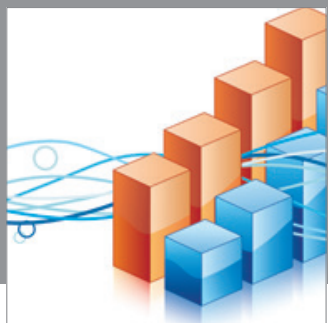

Advances in

Operations Research

mansans

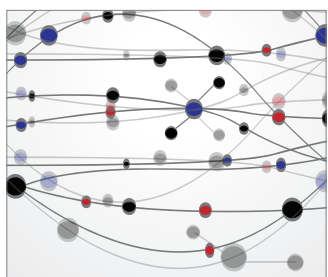

The Scientific World Journal
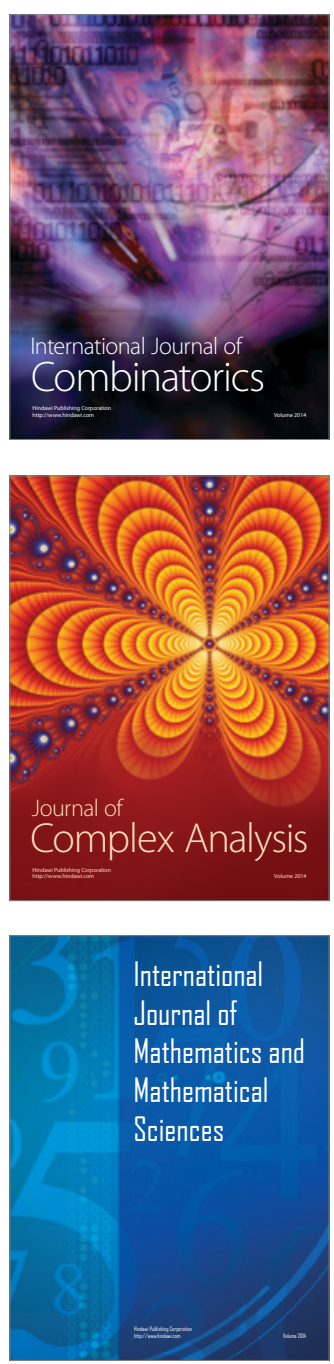
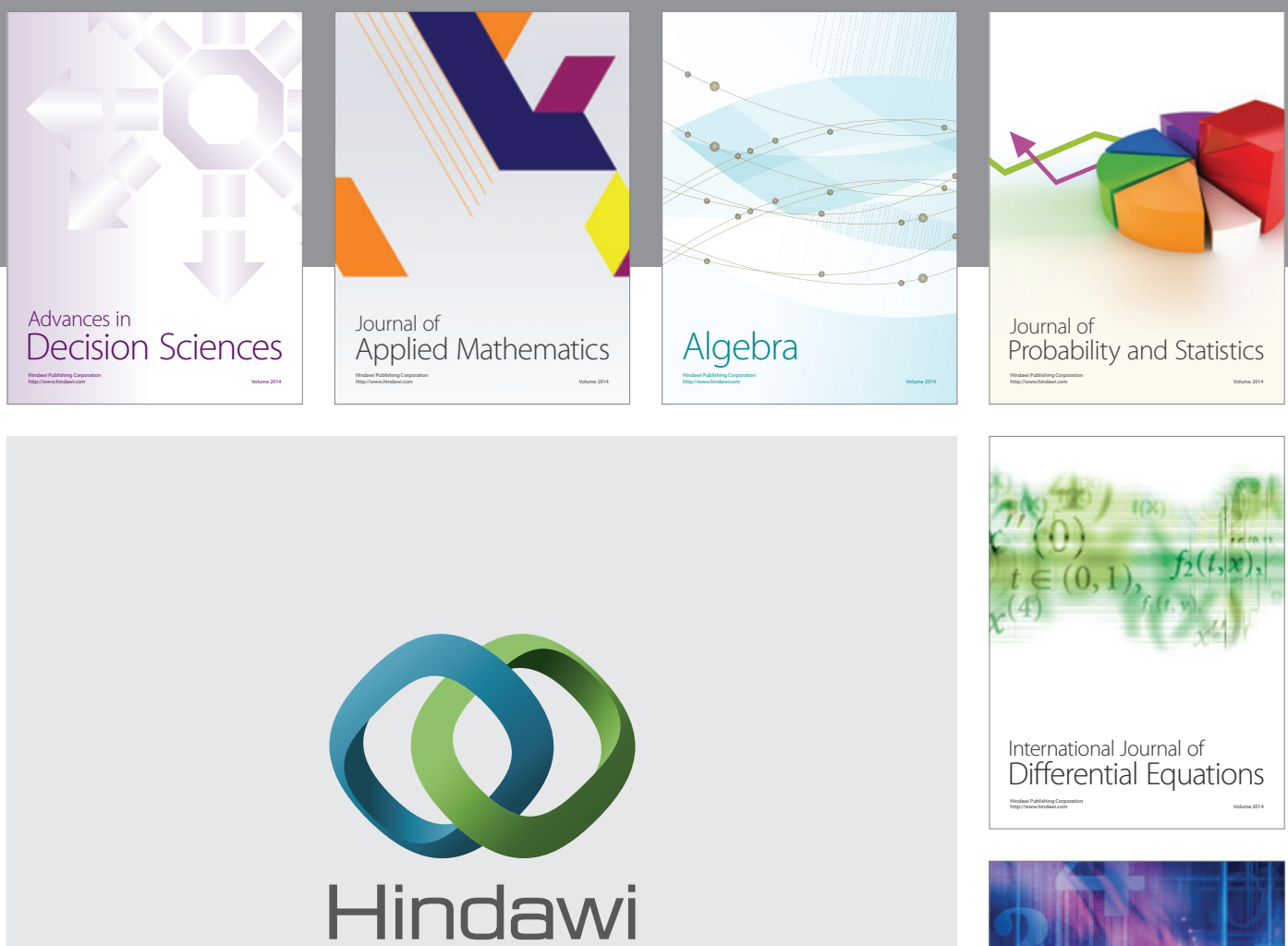

Submit your manuscripts at http://www.hindawi.com
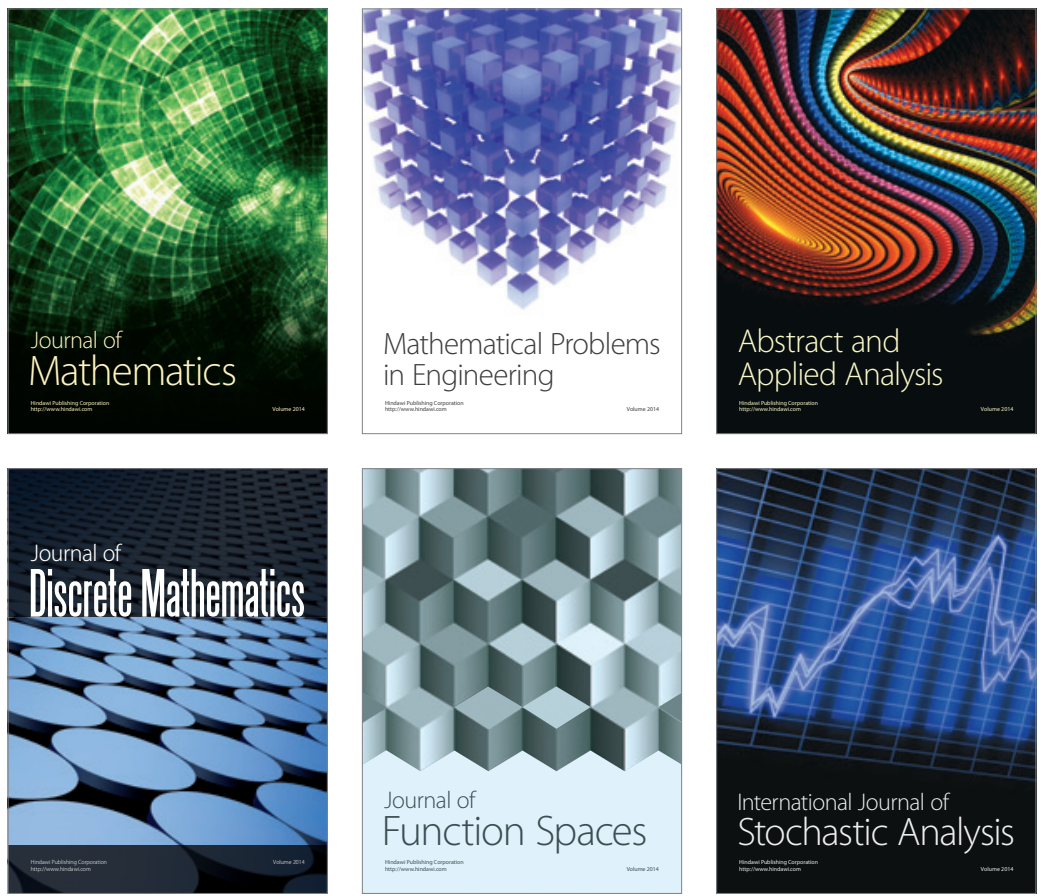

Journal of

Function Spaces

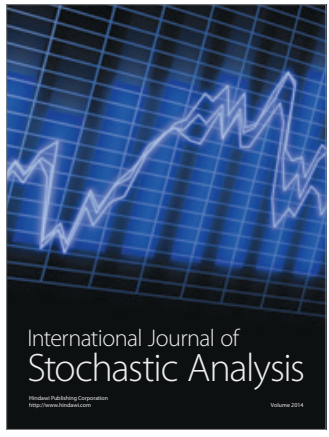

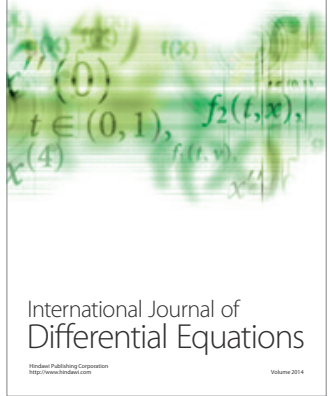
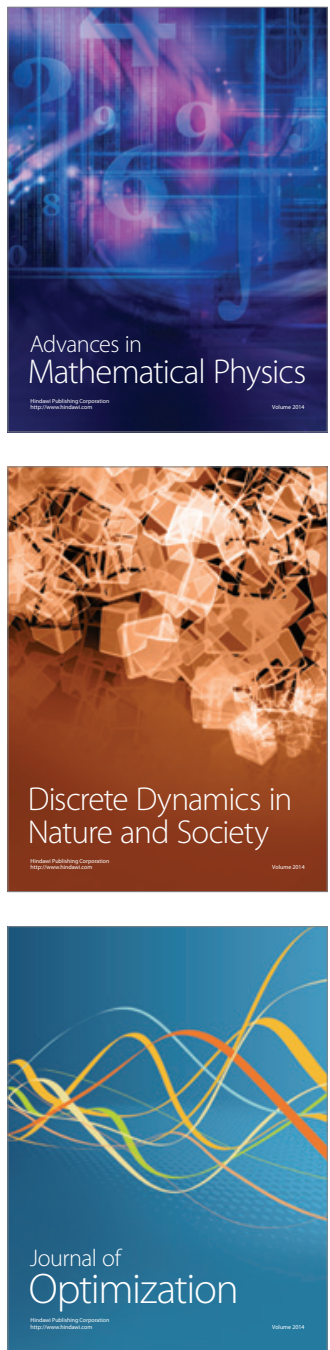